\title{
Imaging Electrochemical Processes in Li Batteries by Operando STEM
}

Nigel D. Browning ${ }^{1,2,3}$, B. Layla Mehdi ${ }^{1,2}$, Andrew Stevens ${ }^{4}$, Wu Xu ${ }^{1,5}$, Wesley A. Henderson ${ }^{5}$, JiGuang Zhang ${ }^{1,5}$, Karl T. Mueller ${ }^{1,2}$, Hardeep Mehta ${ }^{6}$, Libor Kovarik ${ }^{6}$ and Andrey Liyu ${ }^{6}$

1. Joint Center for Energy Storage Research (JCESR), Pacific Northwest National Laboratory (PNNL), Richland, WA, USA

2. Physical and Computational Science Directorate, PNNL, Richland, WA, USA

${ }^{3 .}$ Materials Science and Engineering, University of Washington, Seattle, WA, USA

4. National Security Directorate, PNNL, Richland, WA, USA

${ }^{5 .}$ Energy and Environmental Directorate, PNNL, Richland, WA, USA

6. Environmental Molecular Sciences Laboratory, PNNL, Richland, WA, USA

Reactions at the two interfaces between the main components of a battery - anode/electrolyte and cathode/electrolyte - determine the overall energy density and coulombic efficiency of the system as a whole. While Li-ion batteries are currently the standard for many applications, these batteries have their limitations, and as such, there are research efforts progressing worldwide aimed at developing new more efficient batteries - such as Li-metal, Li-air, Li-sulfur, Na-ion and $\mathrm{Zn}$. In many of these new batteries, the systems that show the most promise in terms of the individual properties of the components run into severe problems when they are combined together to form a full operating cell. Side reactions can cause electrolyte breakdown, passivation or corrosion and the formation of a solid-electrolyte-interphase (SEI) layer. In addition, deposition of an excess of metal ions during charging can lead to the formation of dendrites during cycling. Each of these effects is extremely sensitive to the local chemistry/field at the electrode/electrolyte interface and a full understanding of the materials parameters that can lead to better batteries requires the ability to observe all of the dynamic processes taking place at these interfaces during battery operation.

The kinetics of the reactions at the electrode/electrolyte interface can be studied during battery cycling using in-situ electrochemical stages in the (scanning) transmission electron microscope (S/TEM) [1]. Figure 1 shows the typical field distribution around the Protochips electrochemical stage that allows the fields to be quantified and the most probable location of the reaction to be identified [2]. To make sure that the electron beam does not have any effect on the electrochemistry being performed, the electron beam dose rate is carefully calibrated to be below the electrolyte damage threshold prior to operando electrochemical cycling (in this case all experiments use a dose $\leq 0.3$ electrons $/ \AA^{2} / s$ ) [3]. As such, typical beam effects such as the formation of bubbles and/or precipitates from the breakdown of the electrolyte are completely avoided.

This experimental approach can be used to study, for example, the effect of electrolyte additives on the formation of Li-dendrites (Figure 2) [4]. In this case a $1 \mathrm{M} \mathrm{LiPF} 6$ in propylene carbonate (PC) electrolyte was used with controlled trace-amounts of $\mathrm{H}_{2} \mathrm{O}$ additive (10 and $\left.50 \mathrm{ppm}\right)$. As can be seen clearly from the figure, there is a completely different morphology of the deposits under the two conditions - the low water content shows smaller grains and lower reversibility while the higher water content shows larger grains and higher reversibility (Coulombic efficiency). The reason for this change in properties appears to be related to a reaction between the additive and the electrolyte that gives rise to an increase in the higher conducting LiF inorganic components in the SEI layer - higher conducting channels lead to larger grains. The use of in-situ TEM methods to perform these and other observations from a wide variety of battery systems will be discussed during this presentation [5]. 


\section{References:}

[1] M. Gu et al, Nano Letters 13 (2013), p. 6106.

[2] P. Abellán, et al, Nano Letters 14 (2014), p. 1293.

[3] B. L. Mehdi et al, Nano Letters 15 (2015), p. 2168.

[4] B. Layla Mehdi et al, Scientific Reports 6 (2016), p. 34267.

[5] This work was supported by Joint Center for Energy Storage Research (JCESR), an Energy

Innovation Hub funded by the Department of Energy, Office of Science, Basic Energy Sciences (BES).

The development of the operando stage was supported by the Chemical Imaging Initiative (CII), a

Laboratory Directed Research and Development (LDRD) Program at Pacific Northwest National Laboratory (PNNL). The image analytics used for quantification were developed as part of the Analytics In Motion (AIM) Initiative LDRD Program at PNNL. PNNL is a multi-program national laboratory operated by Battelle for the U.S. Department of Energy (DOE) under Contract DE-AC05-76RL01830. A portion of the research was performed using the Environmental Molecular Sciences Laboratory (EMSL), a national scientific user facility sponsored by the Department of Energy's Office of Biological and Environmental Research and located at PNNL.

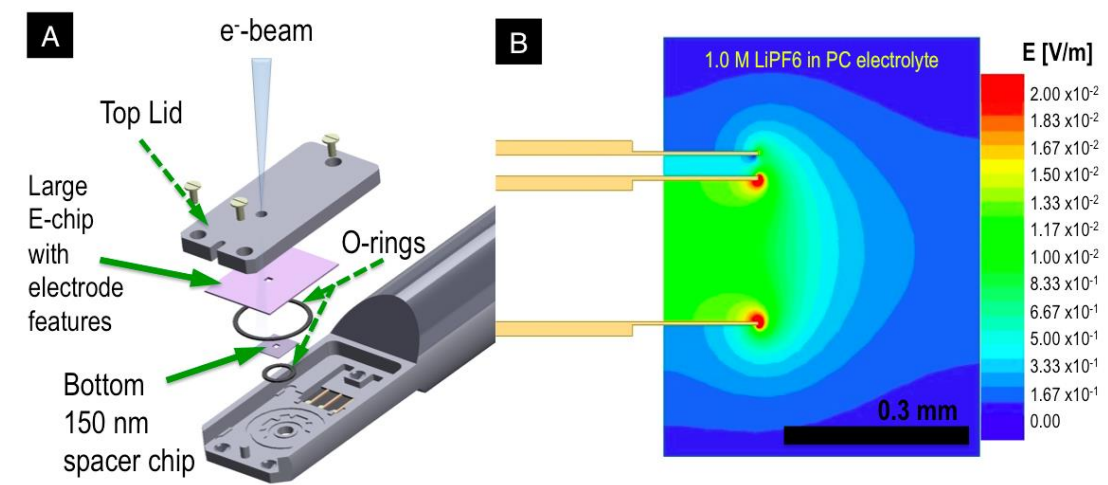

Figure 1. (a) Schematic illustration of the in-situ ec-liquid (S)TEM stage. (b) ANSYS Maxwell static 3D electromagnetic finite element simulation of the electric field distribution in the in-situ liquid (S)TEM cell containing the $\mathrm{LiPF}_{6} / \mathrm{PC}$ electrolyte during galvanostatic discharge at $>0.1 \mathrm{~mA} / \mathrm{cm}^{2}$ with a "hot-spot" localized at the top of the Pt working electrode. The legend shows that during electrochemical cycling the in-situ liquid cell exhibits a non-uniform electric field distribution along the Pt working electrode. However, as Li deposition is observed along the entire length of the Pt electrode, this indicates that a wide range of current densities can be used to initiate the deposition.
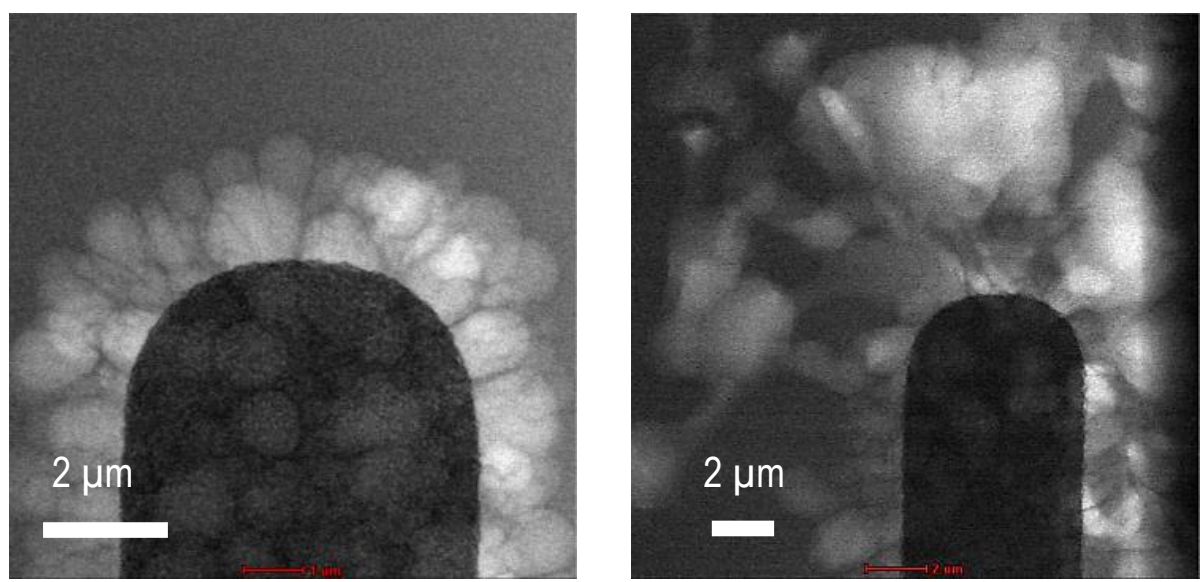

Figure 2. The Li deposit grain size is smaller in the lower water content electrolyte (a) than in the higher water content (b). More Li is deposited and stripped during cycling in (b) resulting in a higher coulombic efficiency. 